\title{
The Linear Frequency Modulation Pulse Signal Adaptive Detection Method
}

\author{
Feng $\mathrm{GaO}^{1, \mathrm{a}}$, Bing Jia ${ }^{1, \mathrm{~b}}$, Guijuan $\mathrm{Li}^{1, \mathrm{c}}$, Mingwei Zhang ${ }^{1, \mathrm{~d}}$ \\ ${ }^{1}$ Science and Technology on Underwater Test and Control Laboratory Dalian 116013, China \\ aemail:285856202@qq.com, bemail:jiabing.robin@gmail.com, cemail:ligj- \\ dl@163.com, demail:17061300@ qq.com
}

Keywords: LFM, pulse compression, correlation peak detection, adaptive detection

\begin{abstract}
It is first elaborated that when the transmission time of underwater acoustic waves is estimated from the transmitting end to the receiving end, LFM pulse signal is often used, because after it is processed through matched filtering, a very important phenomenon will produce, which is pulse compression that a wide pulse in the time dimension is squeezed into a narrow pulse, so that transmission time can be accurately estimated; secondly it is explained that due to the multipath effect in the shallow water, reverberation signal in the propagation process and environmental noise and other factors, when a common detection method of fixed threshold is used, often the wrong detection can be caused, an adaptive detection method is presented for this problem, that is based on the pulse compression of the LFM signal, and the adaptive detection algorithm, the correlation peak of sound waves reach time is detected; finally through processing simulated data and measured data of the sea, it is verified that the this method is effective.
\end{abstract}

\section{Introduction}

It is a commonly used method of underwater acoustic positioning that the beacon is installed in the cooperation of underwater target, using acoustic path difference or phase difference from sound waves to the measuring array to locate[1]. But we know, in the communication process of actual ocean channel, signal detection and processing is a very important step, especially under the circumstances of the presence of multipath effect in shallow water, reverberation signal in the propagation process and the presence of environmental noise, it is very key how to accurately obtain the correlation peak of direct sound. In practical applications LFM signal is often used, we can first use the cross-correlation method of same frequency correlation and different frequency uncorrelation to filter the signal, in order to remove the interference effects of the noise, after signal is processed by matched filtering, a very important phenomenon will produce, that is pulse compression that a wide pulse in the time dimension is squeezed into a narrow pulse, so transmission time can be accurately estimated[2][3]. But in the actual ocean channel it is not easy to effectively detect the correlation peak, using common detection method of fixed threshold, wrong detection is offen generated. So this paper an adaptive detection method is proposed, which can effectively detect the correlation peak. Through the simulation and sea trial data, it is verified that this method is good and has certain practical value.

\section{Analysis of LFM pulse signal characteristics}

Mathematical expression of a linear frequency modulated pulse signal is given in Formula (1)[4], and its waveform is shown in Figure 1.

$$
s_{L F M}(t)=\left\{\begin{array}{cc}
\operatorname{Re}\left[A \exp \left[j 2 \pi\left(f_{L} t+K / 2 * t^{2}\right)\right]\right] & 0<t<T \\
0 & \text { others }
\end{array}\right.
$$




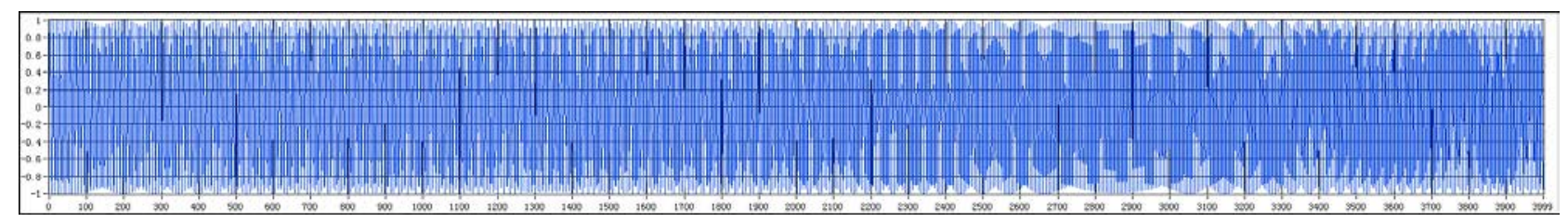

Fig.1 LFM signal time-domain waveform

It is a rectangular pulse that envelope width is $\mathrm{T}$ and frequency linearly changes with time. Among them, $K=\left(f_{L}-f_{H}\right) / T$ is change rate of the signal frequency, or frequency rate, LFM signal FM range is $\left[f_{L}, f_{H}\right]$.

After the LFM pulse signal is treated through self correlation matched filtering, a very important phenomenon will produce, that is pulse compression that a wide pulse in the time dimension is squeezed into a narrow pulse. The following is the analysis of this nature: the envelope of autocorrelation function absolute value of LFM pulse signal is given in Formula (2).

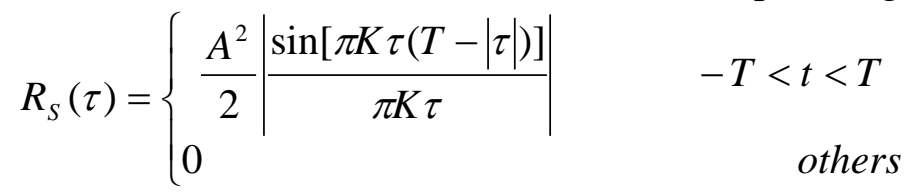

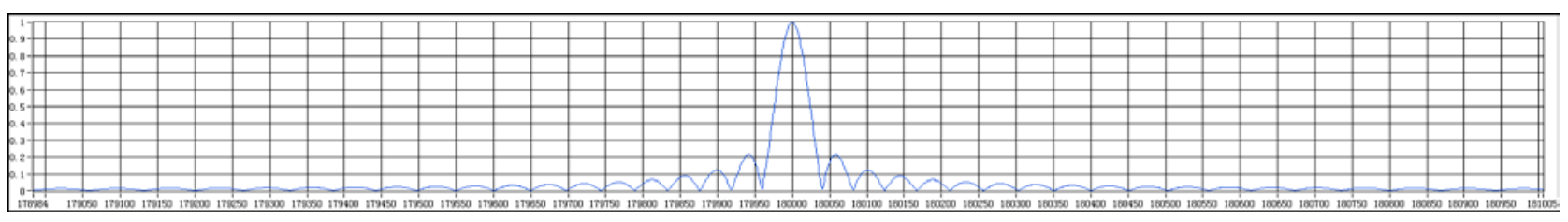

Fig.2 Waveform after the LFM signal is correlation processed

In Figure 2, after LFM signal is processed by matched filtering, the output of its rectangular envelope becomes the $\sin x / x$ function, and it can be seen from (2), when the signal bandwidth $B=f_{L}-f_{H}$ is larger, the main lobe of the correlation function is very narrow, and the mainlobe peak is much higher than the first sidelobe peak, and so easy to detect, so the linear frequency modulated signal has a good correlation, therefore in the estimation of the transmission time of underwater acoustic waves from the transmitting end to the receiving end, this signal is usually used.

\section{Adaptive detection method}

In the common detection methods of fixed threshold, the selected threshold is a preset constant, but this detection method will appear some problems in practical application. On the one hand, if the amplitude of signal is smaller, the correlation peak is smaller, then it is possible that the phenomenon of leak detection will occur because the relevant peak is smaller than the set threshold value; on the other hand, if the noise is increased, so the smaller threshold will cause false alarm phenomenon. From the above analysis it be can seen, it is the shortcomings of fixed threshold method, that the absolute value of correlation peak is related to the size of the received signal, too high easily lead to leak detection increasing, too low easily cause false alarm rate increasing. At the same time, because of the influence of ocean channel, matching peak is more than one, but multiple, and the maximum peak does not always appear in the direct sound arriving time, most often appears in the reflected signal arriving time, so misjudgment is also caused. For these phenomena, here we collectively refer them to as wrong examination phenomenon [5][6] .

In order to solve the above problem, this paper an adaptive detection method for the received signal is proposed, the flow diagram of the method is shown in Figure 3. Firstly, the received signal is processed by matched filtering, then the single received signal is normalized, again using the peak detection method, but this method a fixed threshold and peak width are detected, when detecting a plurality of peak, the corresponding time of the first peak is the arrival time of the sound signal. 


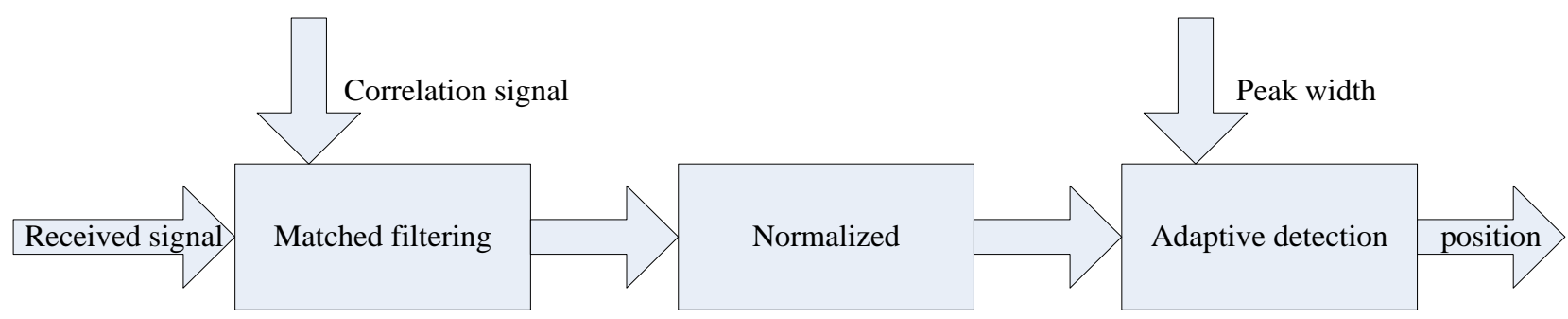

Fig.3 Flow chart of adaptive detection method

\section{Simulation and test results}

To verify the validity of the method, simulation data test and measured data of the sea are processed.

a). laboratory simulation test data

Below the effectiveness of LFM signal is verified through simulation. In Figure 4, signal sampling rate is $\mathrm{FS}=200 \mathrm{KHZ}$, the upper and lower limits of the linear FM signal are respectively $\mathrm{FL}=25 \mathrm{KHZ}$ and $\mathrm{FH}=30 \mathrm{KHZ}$, sweep time is $\mathrm{T}=20 \mathrm{MS}$, it appears at the moment of 0.9 second in 1 second long signals, and peak obtained after pulse compression processing just appears at the moment,as shown in Figure 5, so the effectiveness of LFM using pulse compression processing to obtain the propagation time of the signal can be proved.

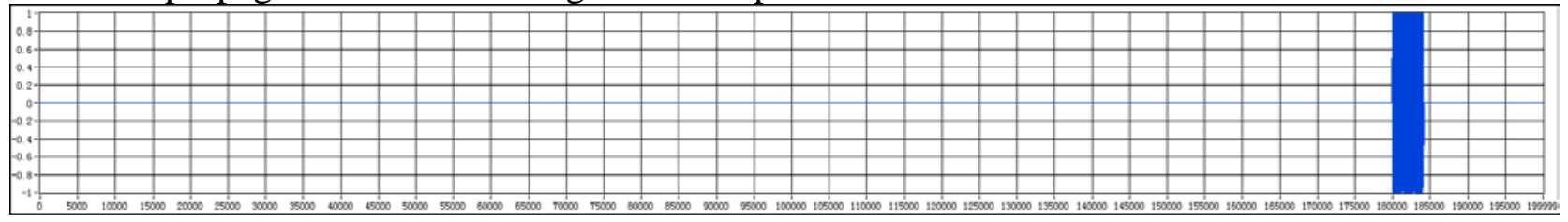

Fig.4 The simulation of signal

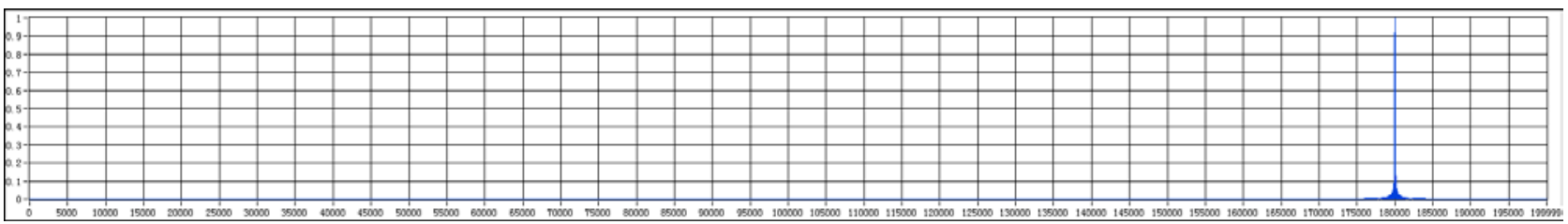

Fig.5 Results after the treatment

b). measured data test of the sea

In order to verify the practicability of the adaptive detection method, a sea test is conducted, that the launch sound source is dipped to the depth of 9 meters underwater and towed by a boat, upper and lower of launch are respectively linear $\mathrm{FM}$ signal $\mathrm{FL}=25 \mathrm{KHZ}$ and $\mathrm{FH}=30 \mathrm{KHZ}$, sweep time is $\mathrm{T}=20 \mathrm{MS}$, each frame interval is $4 \mathrm{~S}$. A receiving hydrophone also is deployed to the depth of 9 meters underwater and fixed, so as to receive signal. In Figure 6 voltage signal received by the hydrophone is shown. Figure 7 and 8 respectively are graphs after two different frame signals are matched, and from the graghs it can be seen that there will be multiple matching peaks because of the influence of ocean channel, sometimes, the direct sound summit is less than the reflected signal, but we need to find the arriving time of the direct sound. In Figure 7 and 8 , the test results are respectively shown after using the matching peak method and adaptive method, and we can see that the correct detection results can be obtained through using the adaptive method. 


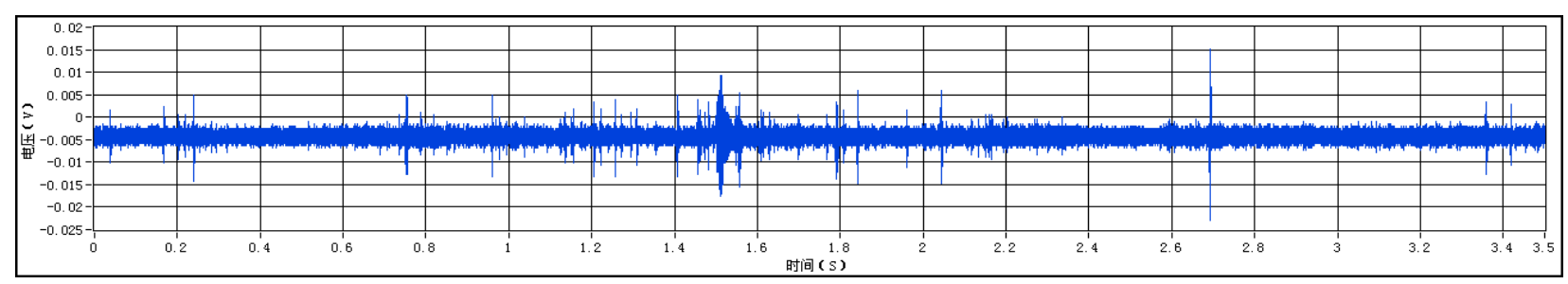

Fig.6 The hydrophone signal

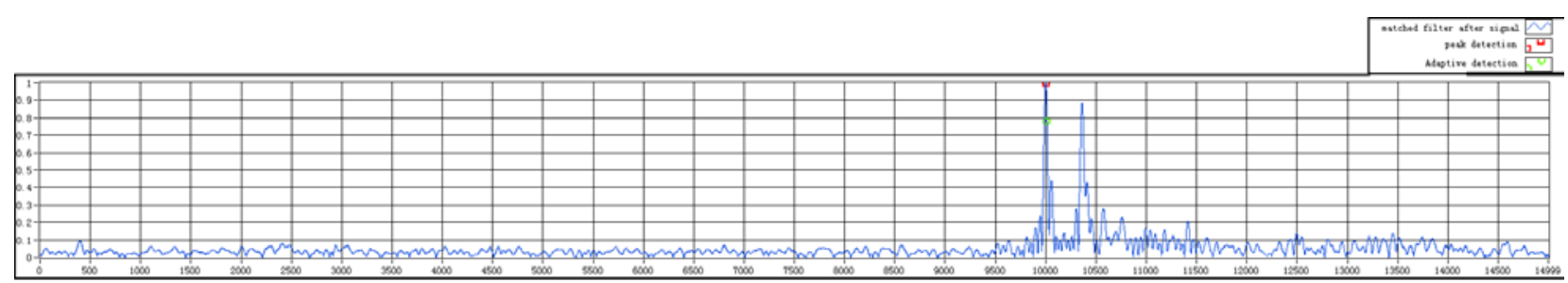

Fig.7 judgment result graph when the direct sound signal is greater than the reflected sound signal

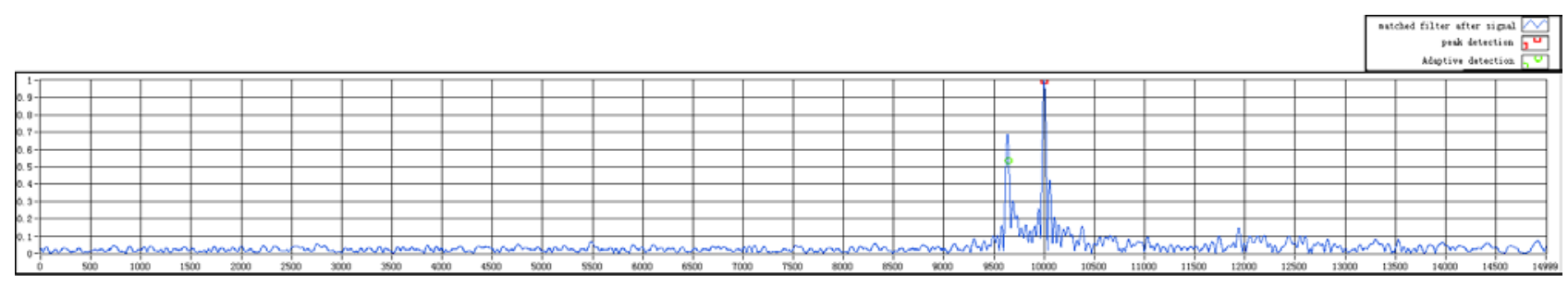

Fig.8 judgment result graph when the direct sound signal is less than the reflected sound signal

During the whole experiment, there are a total of 160 times transmitted signals while moving sound source, and the receiving end is fixed to receive, moving distance of target is as shown in Figure 9. In Figure 10 the comparative error is shown between obtained value by using matching peak detection method and adaptive detection method and GPS truth value.

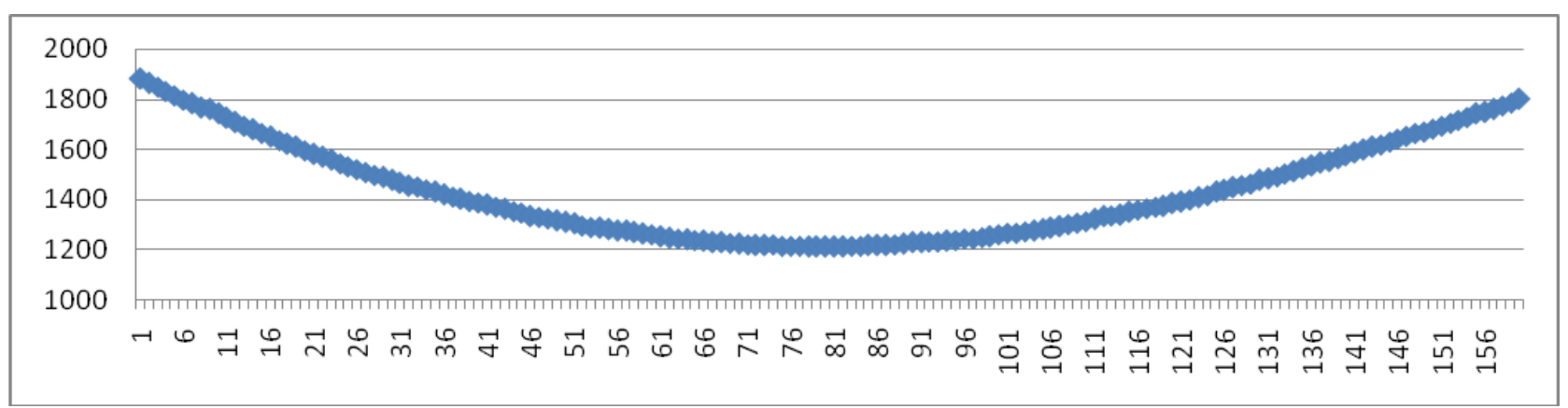

Fig.9 Moving distance of target

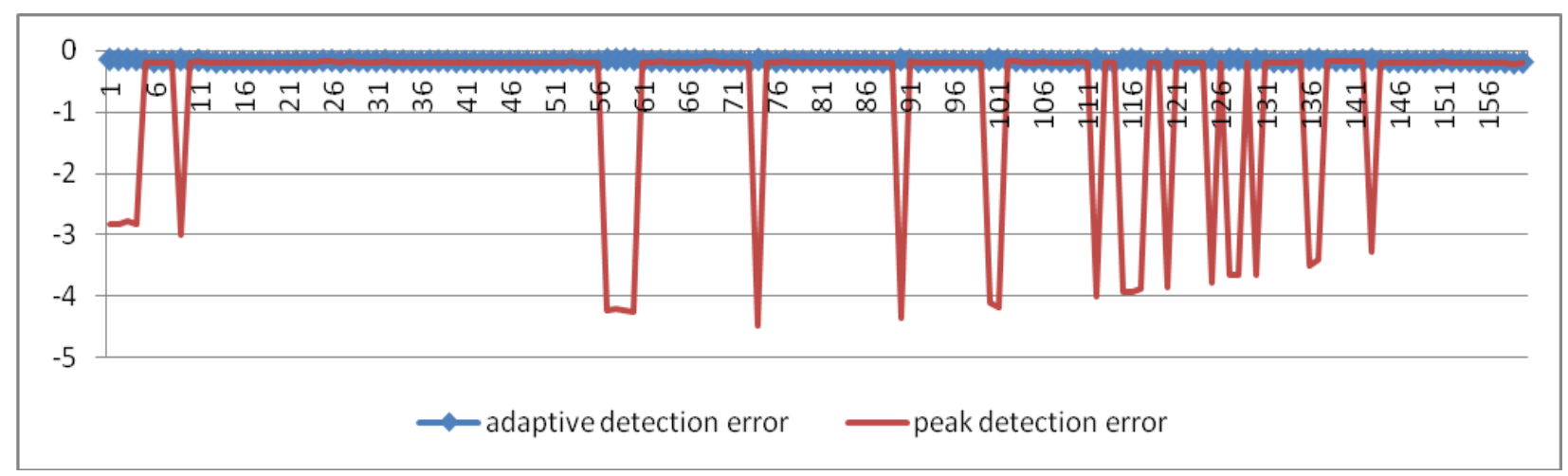

Fig.10 Comparative error 
We can see that $100 \%$ peak correct detection probability can be hitted through using adaptive detection method in the data processing of 160 frames, but using a fixed threshold method it can appear multiple times wrong examination.

\section{Conclusion}

This paper an adaptive detection method is presented, and the experimental results show that the accuracy of detection can be greatly improved through this method, and the probability of failure can be reduced.

\section{References}

[1] Carter G Future Trends in Sonar Signal Procssing[M].Underwater Acoustic Data Processing new London,USA.,1988.203 213.

[2] CHOI K,CHEUN K,JUNG T,Adaptive PN code acquisition using instantaneous power-scaled detection threshold under Rayleigh fading and pulsed Gaussian noise jamming[J].IEEE Transactions on Communications ,2002,50(8):1232-1235.

[3] ZHENG Jihua . Automatic gain control and signals processing technique of underwater ultrasonic receiver[D].Nanjing:Institutes of Technology of Nanjing,2007.

[4] TIAN Tan, LIU Guozhi, SUN Dajun. Techniques of sonar[M].Harbin:Harbin Engineering University Press,2006.

[5] HUI Junying,SHENG Xueli.Underwater acoustic channel[M].Beijing: National Defense Industry Press,2007.

[6] ZHU Changping, HAN Qingbang. Priciples and applications of underwater acoustic communication[M].Beijing: Publishing house of electronics industry,2009. 